\title{
THE JIANG-SU ALGEBRA DOES NOT ALWAYS EMBED
}

\author{
Marius Dadarlat, Ilan Hirshberg, Andrew S. Toms, and Wilhelm \\ WINTER
}

\begin{abstract}
We exhibit a unital simple nuclear non-type-I $\mathrm{C}^{*}$-algebra into which the Jiang-Su algebra does not embed unitally. This answers a question of M. Rørdam.
\end{abstract}

The Jiang-Su algebra, denoted by $\mathcal{Z}([3])$, occupies a central position in the structure theory of separable amenable $\mathrm{C}^{*}$-algebras. The property of absorbing the Jiang$\mathrm{Su}$ algebra tensorially is a necessary, and, in considerable generality, sufficient condition for the confirmation of G. A. Elliott's K-theoretic rigidity conjecture for simple separable amenable $\mathrm{C}^{*}$-algebras $([5],[7])$. The uniqueness question for this algebra is therefore of great interest. M. Rørdam observed that if $\mathcal{C}$ is a class of unital separable $\mathrm{C}^{*}$-algebras, and $A \in \mathcal{C}$ has the properties that (i) for every $B \in \mathcal{C}$ there is a unital *-homomorphism $\gamma: A \rightarrow B$ and (ii) every unital $*$-endomorphism of $A$ is approximately inner, then $A$ is the only such algebra, up to isomorphism. (This follows from an application of Elliott's Intertwining Argument.) Every unital *-endomorphism of $\mathcal{Z}$ is approximately inner ([3]), and there are no obvious obstructions to the existence of a unital $*$-homomorphism $\gamma: \mathcal{Z} \rightarrow A$ for any unital separable $\mathrm{C}^{*}$-algebra $A$ without finite-dimensional quotients. Indeed, such a $\gamma$ always exists when $A$ has real rank zero, and examples show that the existence of $\gamma$ is strictly weaker than tensorial absorption of $\mathcal{Z}$-see [1] and [6], respectively. All of this begs the question, first posed by Rørdam: "Does every unital $\mathrm{C}^{*}$-algebra without finite-dimensional quotients admit a unital embedding of $\mathcal{Z}$ ?", see [1]. We prove that the answer is negative, even when the target algebra is simple and nuclear.

Theorem. There is a unital simple nuclear infinite dimensional $C^{*}$-algebra (in fact, an AH algebra) into which the Jiang-Su algebra does not embed unitally.

In the remainder of the paper we give some background discussion and prove the theorem. For a pair of relatively prime integers $p, q>1$, we set

$$
Z_{p, q}=\left\{f \in \mathrm{C}\left([0,1] ; \mathrm{M}_{p} \otimes \mathrm{M}_{q}\right) \mid f(0) \in \mathrm{M}_{p} \otimes 1, f(1) \in 1 \otimes \mathrm{M}_{q}\right\} .
$$

Each $Z_{p, q}$ is contained unitally in $\mathcal{Z}$. If a unital $\mathrm{C}^{*}$-algebra $A$ admits no unital *-homomorphism $\gamma: Z_{p, q} \rightarrow A$, then there is no unital embedding of $\mathcal{Z}$ into $A$.

Let $A, B$ be unital $\mathrm{C}^{*}$-algebras, and let $e, f \in A$ be projections satisfying

$$
(n+1)[e] \leq n[f]
$$

Received by the editors December 11, 2007. Revision received December 15, 2008.

2000 Mathematics Subject Classification. Primary 46L35, Secondary 46L80.

Key words and phrases. Jiang-Su algebra, embeddability.

The authors were partially supported by the Fields Institute; M.D. was partially supported by NSF grant \#DMS-0500693; I.H. was partially supported by the Israel Science Foundation (grant No. 1471/07); A.T. was partially supported by NSERC. 
in the Murray-Von Neumann semigroup $\mathrm{V}(A)$ for some $n \in \mathbb{N}$. It is implicitly shown in the proof of [4, Lemma 4.3] that if $\gamma: Z_{n, n+1} \rightarrow B$ is a unital $*$-homomorphism, then $\left[e \otimes 1_{B}\right] \leq\left[f \otimes 1_{B}\right]$ in $\mathrm{V}(A \otimes B)$; tensor products are minimal. Example 4.8 of [2] exhibits a sequence $\left(B_{j}\right)_{j \in \mathbb{N}}$ of unital separable $\mathrm{C}^{*}$-algebras with the following property: there are projections $e, f \in B_{1} \otimes B_{2}$ such that $4[e] \leq 3[f]$, but $\left[e \otimes 1_{\bigotimes_{j=3}^{n} B_{j}}\right] \not \subset\left[f \otimes 1_{\bigotimes_{j=3}^{n} B_{j}}\right]$ for any $n \geq 3$. Using Rørdam's result, one concludes that there is no unital $*$-homomorphism $\gamma: Z_{3,4} \rightarrow \bigotimes_{j=3}^{n} B_{j}$ for any $j \geq 3$. (In fact, there is nothing special about $Z_{3,4}$. A similar construction can be carried out for a wide variety of $Z_{p, q}$ s.)

To simplify notation, we renumber the $B_{j}$ s so that $Z_{3,4}$ does not embed into $\bigotimes_{j=1}^{n} B_{j}$ for any $n \in \mathbb{N}$. For each $i \in \mathbb{N}$, set $D_{i}=\bigotimes_{j=1}^{i} B_{j}$. We will perturb the canonical embeddings

$$
\psi_{i}:=\mathrm{id} \otimes 1_{B_{i+1}}: D_{i} \longrightarrow D_{i} \otimes B_{i+1}=D_{i+1}
$$

to maps $\phi_{i}$ with the property that $\left(D_{i}, \phi_{i}\right)_{i \in \mathbb{N}}$ has simple limit $D$. Any such limit, simple or not, fails to admit a unital $*$-homomorphism $\gamma: Z_{3,4} \rightarrow D$, and so also fails to admit a unital embedding of $\mathcal{Z}$. Indeed, suppose that such a $\gamma$ did exist. Then, by the semiprojectivity of $Z_{3,4}([3])$, there would exist a unital $*$-homomorphism $\tilde{\gamma}: Z_{3,4} \rightarrow D_{i}$ for some $i$, contradicting our choice of $D_{i}$. We remark that, in particular, $\bigotimes_{j=1}^{\infty} B_{j}$ admits no unital embedding of $\mathcal{Z}$. This algebra is a continuous field of $\mathrm{C}^{*}$-algebras whose fibres are $\mathcal{Z}$-absorbing - in fact, its fibres are all isomorphic to the CAR algebra (see [2, Example 4.8]).

The $B_{j}$ s have the form $\left(e_{j} \oplus f_{j}\right)\left(\mathrm{C}\left(X_{j}\right) \otimes \mathcal{K}\right)\left(e_{j} \oplus f_{j}\right)$, where $e_{j}$ and $f_{j}$ are rank one projections and $X_{j}=\left(\mathrm{S}^{2}\right)^{\times m(j)}$. Let $\alpha: X_{j} \rightarrow X_{j}$ be a homeomorphism homotopic to the identity map, and view $B_{j}$ as a corner of $\mathrm{C}\left(X_{j}\right) \otimes \mathrm{M}_{n}$ for some sufficiently large $n \in \mathbb{N}$. The map $\alpha$ induces an automorphism $\alpha^{*}$ of $\mathrm{C}\left(X_{j}\right) \otimes \mathrm{M}_{n}, \alpha^{*}(f)=f \circ \alpha$. In general, $\alpha^{*}$ will not carry $B_{j}$ into $B_{j}$, but this can be corrected. Since $\alpha$ is homotopic to the identity, the projection $e_{j} \oplus f_{j}$ is homotopic, and hence unitarily equivalent, to its image under $\alpha^{*}$. If $u$ is a unitary implementing this equivalence, then $\bar{\alpha}:=\left.\left(\operatorname{Ad}(u) \circ \alpha^{*}\right)\right|_{B_{j}}$ is an automorphism of $B_{j}$. For our purposes, the salient property of $\bar{\alpha}$ is this: if $f \in B_{j}$ and $f(x) \neq 0$ for some $x \in X_{j}$, then $\bar{\alpha}(f)\left(\alpha^{-1}(x)\right) \neq 0$.

It remains to construct the $\phi_{i}$, and prove the simplicity of the resulting inductive limit algebra $D$. Let us set $Y_{i}:=\prod_{j=1}^{i} X_{j}$ where $i \in \mathbb{N}$ or $i=\infty$. We endow $Y_{i}$ with the metric $d(x, y)=\sum_{j=1}^{i} 2^{-j} d_{j}\left(x^{j}, y^{j}\right)$ where $d_{j}$ is the canonical metric on $X_{j}$, normalized so that $X_{j}$ has diameter equal to one.

Choose a dense sequence $\left(z_{k}\right)_{k \in \mathbb{N}}$ in $Y_{\infty}$. Fix $z_{0} \in Y_{\infty}$ and for each $k \in \mathbb{N}$ let $\beta_{k}: Y_{\infty} \rightarrow Y_{\infty}$ be a cartesian product of isometries of $X_{j}$ s which are homotopic to the identity and such that $\beta_{k}\left(z_{0}\right)=z_{k}$. Let $\left(\alpha_{k}\right)_{k \in \mathbb{N}}$ be an enumeration of the set $\left\{\beta_{m} \beta_{n}^{-1}: n, m \in \mathbb{N}\right\}$. It is easy to see that for any point $x \in Y_{\infty}$ and any $i \in \mathbb{N}$, the sequence $\left(\alpha_{k}(x)\right)_{k \geq i}$ is dense in $Y_{\infty}$. Note that each $\alpha_{k}$ is also a cartesian product of isometries $\alpha_{k}^{j}$ of $X_{j}$ homotopic to $\operatorname{id}_{X_{j}}$. Let us set $\alpha_{k,[i]}=\prod_{j=1}^{i} \alpha_{k}^{j}$. Let $\pi_{i}: Y_{\infty} \rightarrow Y_{i}$ be the co-ordinate projection. Then $\pi_{i} \alpha_{k}(x)=\alpha_{k,[i]}\left(\pi_{i}(x)\right)$ for $x \in Y_{\infty}$. Therefore for any point $y \in Y_{i}$, the sequence $\left(\alpha_{k,[i]}(y)\right)_{k \geq i}$ is dense in $Y_{i}$. So is the sequence $\left(\left(\alpha_{k,[i]}\right)^{-1}(y)\right)_{k \geq i}$ since each $\alpha_{k,[i]}$ is an isometry. By the compactness of $Y_{i}$ it follows that for any nonempty open set $U$ of $Y_{i}$, there is $j \geq i$ such that $Y_{i}=\bigcup_{k=i}^{j}\left(\alpha_{k,[i]}\right)^{-1}(U)$. 
For each $i \leq k \in \mathbb{N}$, let $\overline{\alpha_{k,[i]}}: D_{i} \rightarrow D_{i}$ be the automorphism induced, in the manner described above, by the homeomorphism $\alpha_{k,[i]}: Y_{i} \rightarrow Y_{i}$.

Observe that the canonical embedding $\psi_{i}: D_{i} \rightarrow D_{i+1}$ is the direct sum of two non-unital embeddings:

$$
\psi_{i}^{(1)} \stackrel{\text { def }}{=} \mathrm{id} \otimes e_{i+1}: D_{i} \rightarrow D_{i} \otimes e_{i+1} \subseteq D_{i+1},
$$

and

$$
\psi_{i}^{(2)} \stackrel{\text { def }}{=} \mathrm{id} \otimes f_{i+1}: D_{i} \rightarrow D_{i} \otimes f_{i+1} \subseteq D_{i+1} .
$$

Set $\phi_{i}^{(1)}=\psi_{i}^{(1)}$, and

$$
\phi_{i}^{(2)} \stackrel{\text { def }}{=} \overline{\alpha_{i,[i]}} \otimes f_{i+1}: D_{i} \rightarrow D_{i} \otimes f_{i+1} \subseteq D_{i+1} .
$$

Define $\phi_{i}: D_{i} \rightarrow D_{i+1}$ to be $\phi_{i}^{(1)} \oplus \phi_{i}^{(2)}$.

Let us now verify that $D=\lim _{i \rightarrow \infty}\left(D_{i}, \phi_{i}\right)$ is simple. It will suffice to prove that for any nonzero $a \in D_{i}$ there is some $j \geq i$ such that $\phi_{i, j+1}(a):=\phi_{j} \circ \cdots \circ \phi_{i}(a)$ is nonzero over every point in the spectrum of $D_{j+1}$.

For each $\mathbf{v}=\left(v_{i}, \ldots, v_{j}\right) \in\{1,2\}^{j-i+1}$, set

$$
\phi_{i, j+1}^{\mathbf{v}}=\phi_{j}^{v_{j}} \circ \phi_{j-1}^{v_{j-1}} \circ \cdots \circ \phi_{i}^{v_{i}},
$$

and note that $\phi_{i, j+1}=\bigoplus_{\mathbf{v} \in\{1,2\}^{j-i+1}} \phi_{i, j+1}^{\mathbf{v}}$. For $k \in\{i, \ldots, j\}$, let $\mathbf{v}_{k} \in\{1,2\}^{j-i+1}$ be the vector which is equal to 1 in each co-ordinate except the $k^{\text {th }}$ one. We have (with the exception of the cases $k=i, j$ when the formula reads slightly differently)

$$
\begin{aligned}
& \phi_{i, j+1}^{\mathbf{v}_{k}}(a)=\left[\overline{\alpha_{k,[k]}}\left(a \otimes e_{i+1} \otimes \cdots \otimes e_{k}\right)\right] \otimes f_{k+1} \otimes e_{k+2} \otimes \cdots \otimes e_{j+1} \\
= & {\left.\left[\overline{\alpha_{k,[i]}}(a) \otimes \overline{\alpha_{k}^{i+1}}\left(e_{i+1}\right) \otimes \cdots \otimes \overline{\alpha_{k}^{k}}\left(e_{k}\right)\right)\right] \otimes f_{k+1} \otimes e_{k+2} \otimes \cdots \otimes e_{j+1} . }
\end{aligned}
$$

Since $a$ is nonzero on some nonempty open set $U$, the formula above shows that $\phi_{i, j+1}^{\mathbf{v}_{k}}(a)$ is nonzero on $W_{i, j+1}^{k}:=\left(\alpha_{k,[i]}\right)^{-1}(U) \times X_{i+1} \times \cdots \times X_{j+1}$, for any $j \geq$ $i$. As noticed earlier, there is $j \geq i$ such that $Y_{i}=\bigcup_{k=i}^{j}\left(\alpha_{k,[i]}\right)^{-1}(U)$. Therefore $\bigoplus_{k=i}^{j} \phi_{i, j+1}^{\mathbf{v}_{k}}(f)$ is nonzero on $\bigcup_{k=i}^{j} W_{i, j+1}^{k}=Y_{j+1}=\widehat{D_{j+1}}$, as required.

\section{References}

[1] G. A. Elliott and M. Rørdam, Perturbation of Hausdorff moment sequences, and an application to the theory of $C^{*}$-algebras of real rank zero, In Operator Algebras, The Abel Symposium 2004. Springer-Verlag (2006), 97-115.

[2] I. Hirshberg, M. Rørdam, and W. Winter, $C_{0}(X)$-algebras, stability and strongly self-absorbing $C^{*}$-algebras, Math. Ann. 339 (2007), 695-732.

[3] X. Jiang and H. Su, On a simple unital projectionless $C^{*}$-algebra, Amer. J. Math. 121 (1999), 359-413.

[4] M. Rørdam, The stable and the real rank of $\mathcal{Z}$-absorbing $C^{*}$-algebras, Internat. J. Math. 15 (2004), no. 10, 1065-1084.

[5] _ A simple $C^{*}$-algebra with a finite and an infinite projection, Acta Math. 191 (2003), 109-142.

[6] A. S. Toms, On the classification problem for nuclear $C^{*}$-algebras, Ann. of Math. (2) 167 (2008), 1059-1074.

[7] W. Winter, Localizing the Elliott conjecture at strongly-self absorbing $C^{*}$-algebras (with appendix by H. Lin), electronic preprint, arXiv: math.OA/0708.0283v3 
Department of Mathematics, Purdue University, 150 N. University St., West Lafayette, IN, 47907-2067, USA

E-mail address: mdd@math.purdue.edu

Department of Mathematics, Ben Gurion University of the Negev, P.O.B. 653, Be'er SHEVA 84105, ISRAEL

E-mail address: ilan@math.bgu.ac.il

Department of Mathematics and Statistics, York University, 4700 Keele St., Toronto, Ontario, Canada, M3J 1P3

E-mail address: atoms@mathstat. yorku.ca

School of Mathematical Sciences, University of Nottingham, University Park, NotTingham, NG7 2RD, United Kingdom

E-mail address: wilhelm.winter@nottingham.ac.uk 